\title{
Multi-spectral vascular oximetry of rat dorsal spinal cord
}

Ochoa-Gutierrez, Victor, Konda, Pavan, Motaghian, Sara, Reboud, Julien, Cooper, Jonathan, et al.

Victor Ochoa-Gutierrez, Pavan C. Konda, Sara Motaghian, Julien Reboud, Jonathan M. Cooper, Andrew R. Harvey, "Multi-spectral vascular oximetry of rat dorsal spinal cord," Proc. SPIE 11230, Optics and Biophotonics in LowResource Settings VI, 1123012 (21 February 2020); doi: 10.1117/12.2558281 


\title{
Multi-spectral vascular oximetry of rat dorsal spinal cord
}

\author{
Victor Ochoa-Gutierrez ${ }^{\mathrm{a}, \mathrm{b}}$, Pavan C. Konda ${ }^{\mathrm{a}}$, Sara Motaghian ${ }^{\mathrm{a}}$, Julien Reboud ${ }^{\mathrm{b}}$, Jonathan M. \\ Cooper $^{\mathrm{b}}$, and Andrew R. Harvey ${ }^{\mathrm{a}}$ \\ aSchool of Physics and Astronomy, University of Glasgow, Glasgow, UK \\ b James Watt School of Engineering, University of Glasgow, Glasgow, UK
}

\begin{abstract}
We describe a visible-light multi-spectral system for vascular oximetry studies that can be implemented in lowand middle-income countries, using a low-cost electronics and optical elements, for instance a Raspberry $\mathrm{Pi}$, a Pi camera under a resolution of 5-megapixel, and four light sources at $480 \mathrm{~nm}, 532 \mathrm{~nm}, 593 \mathrm{~nm}$ and $610 \mathrm{~nm}$. It is designed to quantify the vascular oxygen saturation of the rat dorsal spinal cord, Python custom application synchronizes all elements to execute the imaging process in one system. The device is suitable offers a replacement for high cost bulky systems in drug discovery, tracking disease progression and understanding of progressive and degenerative diseases.
\end{abstract}

Keywords: Multispectral Imaging, Oximetry, Imaging System, Gradient-Index Lenses, Low-resource Settings, Biomedical Engineering.

\section{INTRODUCTION}

The aim of this visible-light multi-spectral system is to achieve a solution capable of multispectral imaging analysis in real time that potentially can be adapted or modified according the especial needs of a particular research and researchers in low- and middle-income countries or remote places, by simply using a rechargeable battery pack to power the portable imaging system. In-vivo microscopic imaging techniques $[1,2]$, i.e. oximetry on mice and rats is widespread and increasingly animal models are used in biomedicine, for drug discovery and tracking disease progression. The number of animals used for experimental purposes continues to rise every year, in 2018 an estimated of 3.6 million procedures were made using mice and rats [3]. We propose an in-vivo imaging system that has the promise to reduce the number of animals by collecting of high-resolution imaging data in longitudinal repeated-measures during one single study especially the oxygen consumption in deep tissue that has been proved that has a relationship to different diseases. ${ }^{4-9}$

\section{LOW-COST SYSTEM OVERVIEW}

An imaging system was assembled and designed using; red LED (625nm), amber LED (590nm), green LED $(525 \mathrm{~nm})$ and blue LED $(475 \mathrm{~nm})$, dichroic optical blocks (Thorlabs - CM1-DCH/M), longpass dichroic mirrors (Thorlabs), focusing lenses, heat sinks (Figs. 1, 2 and 3). Universal port that holds a GRIN lens (GRINtech), structured illumination is used by a fiber-array adapted to Raspberry Pi camera, these elements were used to build a low-cost system for imaging. ${ }^{10}$ In addition, the system is capable of directing the illumination into an annular illumination pattern, allowing a single pass illumination of the vasculature, ${ }^{7}$ i.e. reducing the complexity of the oxygenation measurement in deep tissue and blood vessels for a better understanding of axonal remyelination, multiple sclerosis research, immune cells in lymph nodes and towards a cure for rheumatoid arthritis. The universal imaging sensor; is constituted by a Pi camera (resolution 5-megapixel, 2592x1944-pixel static images) and 3D printed holder for the GRIN lens and the illumination fibers, with a locking mechanism to improve coupling between the camera and the GRIN lens, see Fig. 1. The system uses a Python custom application to control the imaging and illumination elements synchronizing all elements at once for oximetry measurement prospectuses.

Further author information: (Send correspondence to A.R.H.)

A.R.H.: E-mail: Andy.Harvey@glasgow.ac.uk

Optics and Biophotonics in Low-Resource Settings VI, edited by David Levitz, Aydogan Ozcan, Proc. of SPIE Vol. 11230, 1123012 • @ 2020 SPIE · CCC code: 1605-7422/20/\$21 · doi: 10.1117/12.2558281 


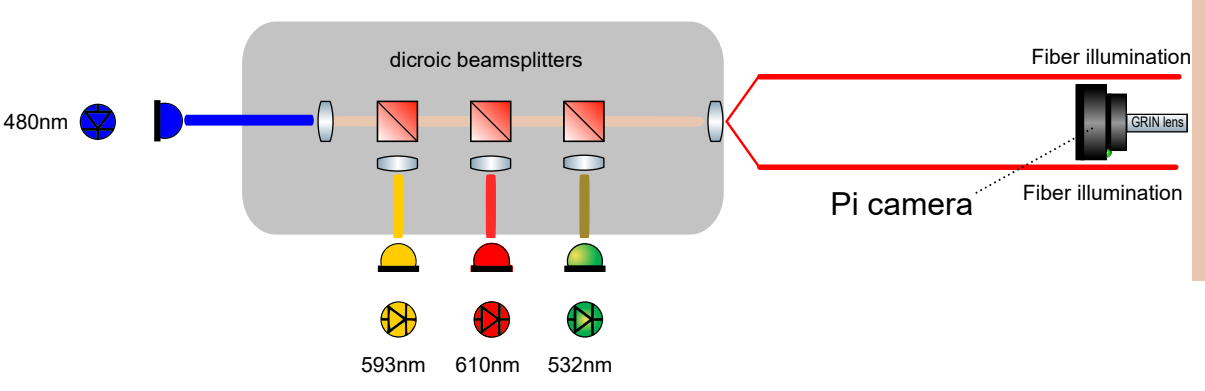

Tissue or sample

Figure 1. Principal imaging system elements; optical illumination, image acquisition and optical magnification using a GRIN lens, tissue representation.
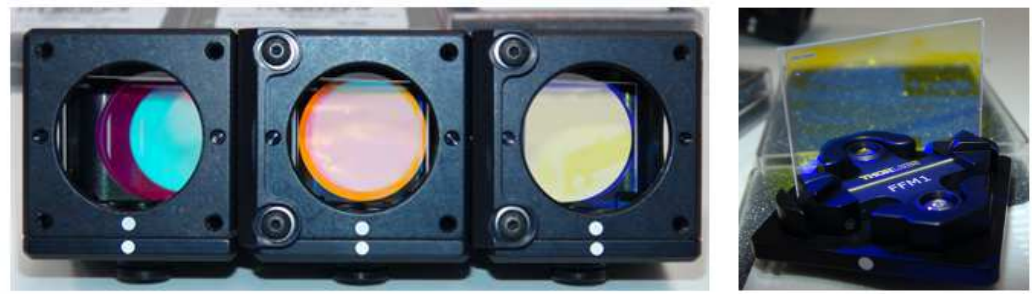

Figure 2. Optical block for structured illumination, dichroic mirrors in position (Thorlabs).

\section{RESEARCH APPLICATIONS FOR IMAGING TECHNIQUES IN LOW-RESOURCE SETTINGS}

The developed system will enable a longitudinal vascular oximetry study using a structured illumination technique on a single animal over a period of four or six weeks. An important contribution is the reduction of use of rodents by a factor four or five. a second important benefit is the reduction of variability inherent in longitudinal study of one single animal, this will enable high quality data related to the degenerative diseases reducing the uncertainty of using more rodents in short periods of time.
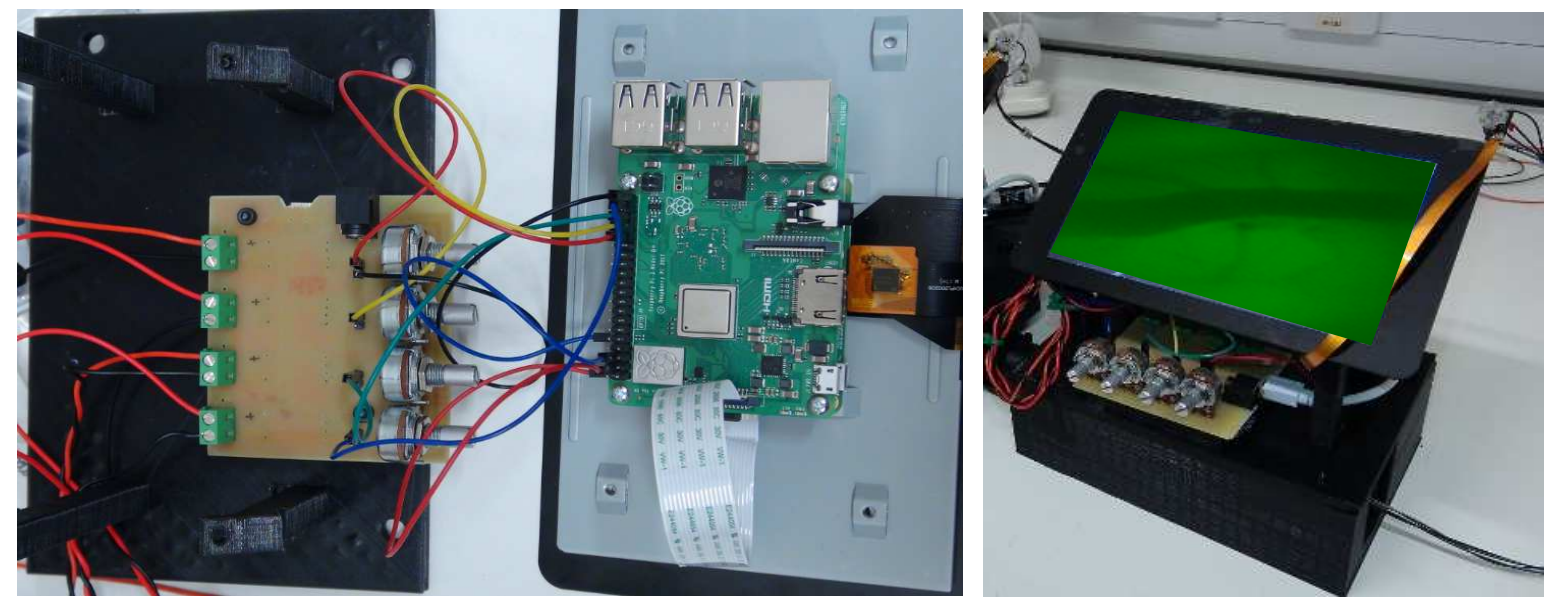

Figure 3. Imaging System completely assembled and operational for research studies. 


\section{INITIAL DEMONSTRATIONS}

Recent evidence has implicated mitochondrial dysfunction, and hypoxia, in the aetiology and pathology of multiple sclerosis (MS) and experimental autoimmune encephalomyelitis, an animal model of MS. ${ }^{6,7}$ The role of these pathological changes in neurological dysfunction, lesion formation, and disease progression remains unclear. However, obvious differences in the vasculature (such as diameter, and oximetry variations, especially hypoxia patterns) have been observed that correlate with measures of disease severity and neurological dysfunction in rats with experimental autoimmune encephalomyelitis (EAE) during the early stages of disease. For preliminary purposes images were recorded from a hyperspectral image sequence of the exposed rat spinal cord shown in Fig. 4. This initial demonstration shows the optical and imaging performance of the system, that provides the vital information for the oximetry signal extraction.
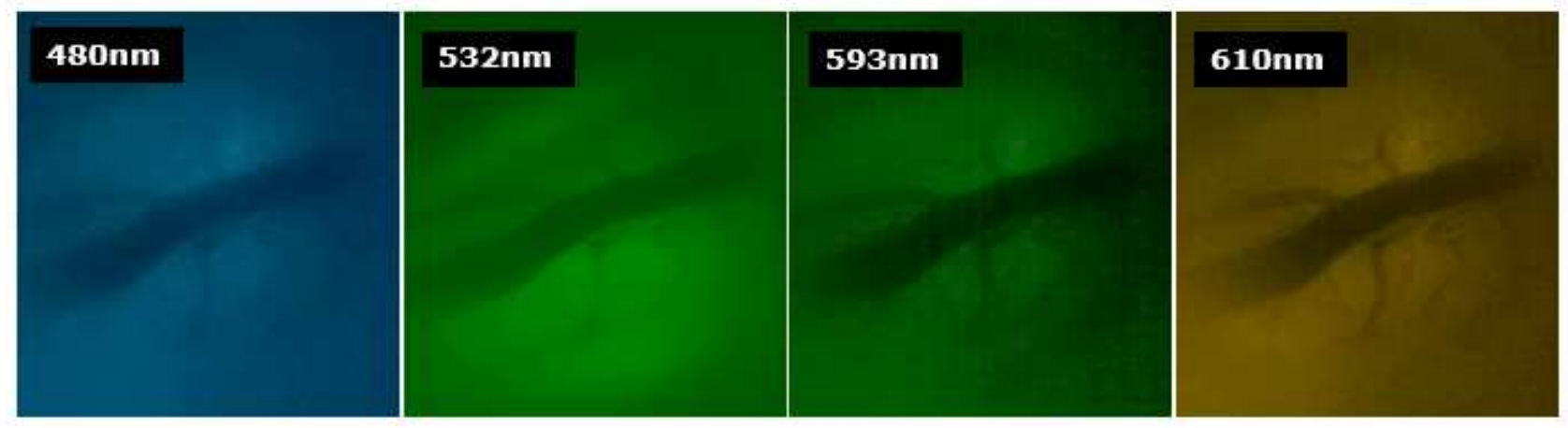

Figure 4. Rat spinal cord vasculature imaged at $480 \mathrm{~nm}, 532 \mathrm{~nm}, 593 \mathrm{~nm}$ and $610 \mathrm{~nm}$. Oximetry is based on varying intensity of blood vessels with wavelength where the changes on oxi-haemoglobin can be measure.

\section{CONCLUSION}

We described a visible-light multi-spectral microscope system for longitudinal oximetry studies in small animals. This device will continue to be improved and continue tested in the field, that includes modifications at the mathematical model establish by $[7,11]$ for oximetry calculations, avoiding some errors when using two oximetry wavelengths $[12,13]$ for multi imaging vasculature studies. Enabling a low-resource settings tool that will lead to better understanding of the progression of degenerative diseases based on the oxygenation levels, especially during the absence of oxygenation or hypoxia stages [14] .

\section{ACKNOWLEDGMENTS}

We are grateful for our funding sources. This research is supported by NC3Rs, IAA-EPSRC, CONACyT-SENER.

\section{REFERENCES}

[1] Desjardins, M., Sylvestre, J.-P., Jafari, R., Kulasekara, S., Rose, K., Trussart, R., Arbour, J. D., Hudson, C., and Lesage, F., "Preliminary investigation of multispectral retinal tissue oximetry mapping using a hyperspectral retinal camera," Experimental Eye Research 146, 330 - 340 (2016).

[2] Rouse, A. R., Udovich, J. A., and Gmitro, A. F., "In-vivo multi-spectral confocal microscopy," (2005).

[3] "Animal research numbers in 2018 — understanding animal research — understanding animal research." http://www.understandinganimalresearch.org.uk/news/communications-media/ animal-research-numbers-in-2018/. (Accessed on 01/07/2020).

[4] Davies, A. L., Desai, R. A., Bloomfield, P. S., McIntosh, P. R., Chapple, K. J., Linington, C., Fairless, R., Diem, R., Kasti, M., Murphy, M. P., and Smith, K. J., "Neurological deficits caused by tissue hypoxia in neuroinflammatory disease," Annals of Neurology 74(6), 815-825 (2013). 
[5] Dyson, A., Stidwill, R., Taylor, V., and Singer, M., "Tissue oxygen monitoring in rodent models of shock," American Journal of Physiology-Heart and Circulatory Physiology 293(1), H526-H533 (2007). PMID: 17384135.

[6] Mahad, D. J., Ziabreva, I., Campbell, G., Lax, N., White, K., Hanson, P. S., Lassmann, H., and Turnbull, D. M., "Mitochondrial changes within axons in multiple sclerosis," Brain 132, 1161-1174 (03 2009).

[7] van der Putten, M. A., MacKenzie, L. E., Davies, A. L., Fernandez-Ramos, J., Desai, R. A., Smith, K. J., and Harvey, A. R., "A multispectral microscope for in vivo oximetry of rat dorsal spinal cord vasculature," Physiological Measurement 38, 205-218 (jan 2017).

[8] Vogel, A., Chernomordik, V. V., Riley, J. D., Hassan, M., Amyot, F., Dasgeb, B., Demos, S. G., Pursley, R., Little, R., Yarchoan, R., Tao, Y., and Gandjbakhche, A. H., "Using noninvasive multispectral imaging to quantitatively assess tissue vasculature," Journal of Biomedical Optics 12(5), 1 - 13 (2007).

[9] Mansfield, J. R., "Multispectral imaging: A review of its technical aspects and applications in anatomic pathology," Veterinary Pathology 51(1), 185-210 (2014). PMID: 24129898.

[10] Fereidouni, F., Griffin, C., Todd, A., and Levenson, R., "Multispectral analysis tools can increase utility of RGB color images in histology," Journal of Optics 20, 044007 (mar 2018).

[11] van der Putten, M. A., Brewer, J. M., and Harvey, A. R., "Multispectral oximetry of murine tendon microvasculature with inflammation," Biomed. Opt. Express 8, 2896-2905 (Jun 2017).

[12] Rodriguez, D. A., Pfefer, T. J., Wang, Q., Lopez, P. F., and Ramella-Roman, J. C., "A Monte Carlo Analysis of Error Associated With Two-Wavelength Algorithms for Retinal Oximetry," Investigative Ophthalmology E Visual Science 57, 6474-6481 (11 2016).

[13] Hashimoto, R., Kurata, T., Sekine, M., Nakano, K., Ohnishi, T., and Haneishi, H., "Two-wavelength oximetry of tissue microcirculation based on sidestream dark-field imaging," Journal of Biomedical Optics 24(3), $1-8(2018)$.

[14] Shi, Y., Oeh, J., Hitz, A., Hedehus, M., Eastham-Anderson, J., Peale, F. V., Hamilton, P., O'Brien, T., Sampath, D., and Carano, R. A., "Monitoring and targeting anti-vegf induced hypoxia within the viable tumor by 19f-mri and multispectral analysis," Neoplasia 19(11), 950 - 959 (2017). 